\title{
Theory of initial stage of the breakdown in non-uniform gas flow
}

\author{
V.E. Semenov ${ }^{1}$, E.I. Rakova ${ }^{1}$, M.Yu. Glyavin ${ }^{1}$, G.S. Nusinovich ${ }^{2}$ \\ ${ }^{1}$ Institute of Applied Physics, Russian Academy of Sciences, Nizhny Novgorod, Russia, \\ ${ }^{2}$ IREAP, University of Maryland, College Park, Maryland 20742-3511, USA \\ sss@appl.sci-nnov.ru
}

In Refs. [1-3] it was suggested to use discharge in Sharply focused microwave beam to create the point-like source of extreme ultraviolet radiation. To reduce the plasma volume in experiments the discharge was ignited in gas flow from small opening into vacuum. In this case the gas density was well localized around the opening. Below the initial stage of the microwave breakdown in non-uniform gas flow was simulated numerically to understand a possibility to localize the discharge plasma and a dependence of the plasma parameters on microwave power and frequency, diameter of the opening and a value of gas pressure, $p_{0}$, at the opening cross section. For simplicity the axially symmetrical problem was considered. Within this model the sharply focused microwave beam was approximated as converging cylindrical TE wave. A dependence of the gas pressure, $p$, on radial coordinate was approximated by the following function

$$
p(r)=\frac{p_{0} a}{\sqrt{a^{2}+r^{2}}}
$$

were $a$ stands for characteristic length of the gas density localization. It was assumed that plasma density is small compared to density of neutral molecules and therefore the ionization process does not affect the gas density distribution. The monochromatic approximation was used to describe electric field amplitude, whereas an evolution of the plasma electron density was simulated taking into account the electron impact ionization, attachment of electrons to neutral molecules and plasma diffusion. Using the normalized variables and parameters the main equations can be presented as follows:

$$
\begin{gathered}
\frac{1}{\rho} \frac{\partial}{\partial \rho}\left(\rho \frac{\partial F}{\partial \rho}\right)+\varepsilon F=0 \\
\frac{\partial n}{\partial \tau}=\frac{1}{\rho} \frac{\partial}{\partial \rho}\left(\rho \frac{1}{P} \frac{\partial n}{\partial \rho}\right)+\mu P n\left[A^{\beta}\left(\frac{1+\delta_{0}^{2}}{1+\delta_{0}^{2} P^{2}}\right)^{\beta / 2}-1\right]
\end{gathered}
$$

Here $F(\rho, \tau)$ stands for complex amplitude of electric field, normalized to the threshold value at opening output, $\rho=\omega r / c$ is radial coordinate, normalized to the wave number of electromagnetic field in vacuum, $\varepsilon=1-n-i \delta n$ is complex dielectric permittivity of plasma, $n=N / N_{c}\left(1+\delta^{2}\right)$ is normalized plasma density, $N_{c}$ denotes the critical plasma density, $\delta=v / \omega=\delta_{0} p / p_{0}$ denotes the normalized frequency, $v$, of electron collisions with neutral molecules, $\tau=D_{0}(\omega / c)^{2} t$ stands for the normalized time, $D_{0}$ is electron diffusion coefficient at the opening output, $P=p / p_{0}$ stands for the normalized gas density, $A=|F|$. Previously the very similar problem was considered in [4] where the breakdown evolution in the converging cylindrical wave taking spatially uniform gas distribution. In this case the numerical simulations shown relatively fast establishment of the stationary plasma density. For the discharge in air with relatively high density (when electron collision frequency exceeds field frequency) the stationary spatial distribution of plasma density was found to be approximately the same as predicted by V.B. Gildenburg on the base of a very simple analytical model [5]. Specifically it was found that an increase in the amplitude of the incident microwave is not accompanied by plasma density at the axis instead this increase results in an increase of the volume occupied by plasma. The plasma volume and density are important parameters when the point like source of extreme UV radiation is under discussion. Therefore a dependence of these parameters on microwave frequency and amplitude, gas nonuniformity and density was under particular interest in our simulations. The simulations were completed within wide range of the gas output pressure, $p_{0}$, length of the gas density non-uniformity, $a$, microwave field frequency and amplitude of the converging cylindrical wave. In all simulations it was found that stationary distribution of plasma density is established relatively fast after the breakdown. Specifically in case of relatively low output density in the gas flow (when the corresponding frequency of electron collisions is small compared to the field frequency, i.e. $\delta_{0}<<1$ ) the plasma density is localized in a region which radius is small compared to electromagnetic wavelength if even a length of the gas flow nonuniformity is not so small (see Fig. 1). In this case a decrease in the amplitude of the converging cylindrical wave is accompanied by a decrease in plasma density whereas spatial distribution of plasma density remains almost unchanged (cf. Fig. 1 and 2)
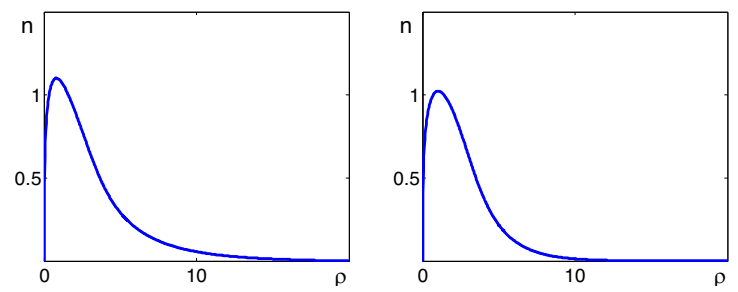

Fig. 1. Spatial distribution of stationary plasma density obtained at high value of normalized amplitude of incident electromagnetic wave $\left(A_{0}=4\right)$ and relatively rarified gas density at the opening output $\left(\delta_{0}=0.3\right.$ ). The left panel presents simulation results obtained with $k a=1$, the right panels presents simulation results with $k a=3$

On the contrary, in case of higher output density in the gas flow (when the corresponding frequency of electron collisions is big compared to the field frequency, i.e. $\left.\delta_{0}>>1\right)$ an increase in the amplitude of incident electromagnetic wave is accompanied by an increase in the vo- 
lume occupied by plasma which is especially pronounced at smaller length of gas density non-uniformity (see Fig. 3).
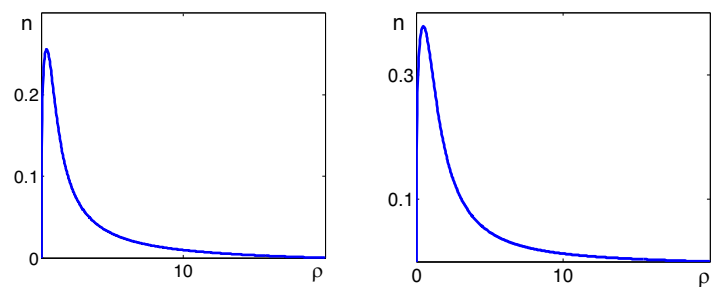

Fig. 2. Similar as in Fig 1 but calculated for less field amplitude in the incident electromagnetic wave $\left(A_{0}=2\right)$
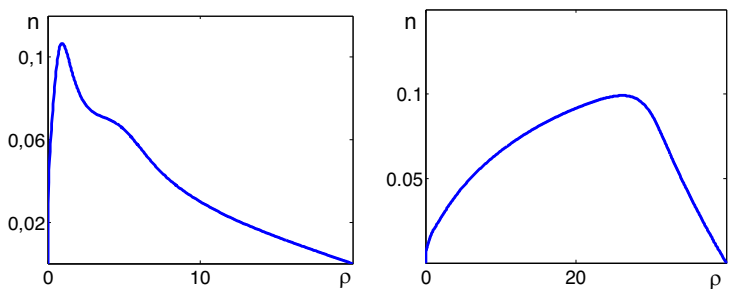

Fig. 3. Spatial distribution of stationary plasma density obtained at relatively high gas density at the opening output $\left(\delta_{0}=3\right)$ and small length of gas non-uniformity $k a=0.3$. The left panel presents simulation results obtained at smaller amplitude of incident electromagnetic wave $\left(A_{0}=2\right)$. The right panel presents simulation results obtained at $A_{0}=4$

On the contrary, in case of higher output density in the gas flow (when the corresponding frequency of electron collisions is big compared to the field frequency, i.e. $\left.\delta_{0}>>1\right)$ an increase in the amplitude of incident electromagnetic wave is accompanied by an increase in the volume occupied by plasma which is especially pronounced at smaller length of gas density non-uniformity (see Fig. 3). In the latter case an increase in the amplitude of incident wave results not only in considerable increase in plasma volume (which can exceed considerably the cube of wavelength) but also in a shift of plasma density maximum to a periphery of plasma. At higher value of $a$, one can find that plasma density distribution in space has two maxima (one in the center and another one is at the periphery of the ionized region see Figs. 4 and 5).

In this case an increase in the incident wave amplitude is accompanied mainly by an increase in the energy deposition at the periphery of ionized region which is not beneficial for creation of the point-like source of extreme ultraviolet radiation.
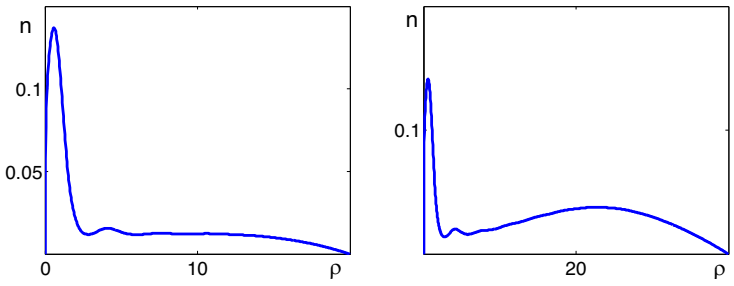

Fig. 4. Similar to Fig. 3, but calculations are completed with $k a=0.3$
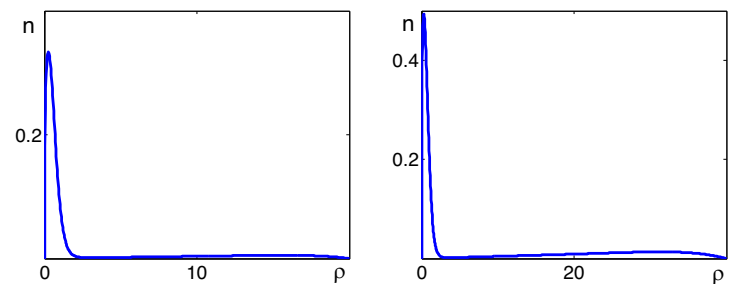

Fig. 5. Similar to Figs. 3-4, but calculations are completed with $k a=3$

\section{Acknowledgements}

The work was supported by the Russian Science Foundation, project No. 14-12-00609.

\section{References}

1. M.Yu. Glyavin, S.V. Golubev, V.G. Zorin, I.V. Izotov, A.G. Litvak, A.G. Luchinin, M.V. Morozkin, S.V. Razin, A.V. Sidorov, V.A. Skalyga. The Discharge Maintained by HighPower Terahertz Radiation in a Nonuniform Gas Flow // Radiophysics and Quantum Electronics. 2014, V.56, No.8-9, P.561-565.

2. M.Yu. Glyavin, S.V. Golubev, I.V. Izotov, A.G. Litvak, A.G. Luchinin, S.V. Razin, A.V. Sidorov, V.A. Skalyga, A.V. Vodopyanov. A point-like source of extreme ultraviolet radiation based on a discharge in a non-uniform gas flow, sustained by powerful gyrotron radiation of terahertz frequency band // Applied Physics Letters, 2014, V.105, P. 174101.

3. A. Sidorov, S. Razin, S. Golubev, M. Safronova, A. Fokin, A. Luchinin, A. Vodopyanov, M. Glyavin. Measurement of plasma density in the discharge maintained in a nonuniform gas flow by a high-power terahertz-wave gyrotron // Phys. Plasmas. 2016, V.23, P.043511.

4. I.B. Bokolishvili, A.V. Kim, G.G. Malinetsky and A.S. Petrosyan. Numerical Simulation of a Nonequilibrium HighFrequency Discharge in a Converging Cylindrical Wave // Physica Scripta. 1990, V.42, No.4, P.442 - 448.

5. V.B. Gil'denburg. Electrodynamic mechanisms that limit the electron concentration in a laser spark // Sov. Phys. JETP, 1980, V.51, No.3, P.480-482. 